\title{
Structural basis for selectivity in flavin-dependent monooxygenase-catalyzed oxidative dearomatization
}

\author{
Attabey Rodríguez Benítez, ${ }^{1}$ Sara Tweedy, ${ }^{1}$ Summer A. Baker Dockrey, ${ }^{2}$ April L. Lukowski, ${ }^{1}$ Troy Wymore, ${ }^{3}$ Dheeraj
} Khare, ${ }^{4}$ Charles L. Brooks III, ${ }^{1,2,3}$ Janet L. Smith, ${ }^{1,5,6}$ Alison R. H. Narayan ${ }^{1,2,6 *}$

\author{
Affiliations \\ ${ }^{1}$ Program in Chemical Biology, University of Michigan, Ann arbor Michigan 48109 \\ ${ }^{2}$ Department of Chemistry, University of Michigan, Ann Arbor, Michigan 48109 \\ ${ }^{3}$ Department of Biophysics, University of Michigan, Ann Arbor Michigan, 48109 \\ ${ }^{4}$ IDEXX Westbrook, Portland ME, 04092 \\ ${ }^{5}$ Department of Biological Chemistry, University of Michigan, Ann Arbor Michigan 48109 \\ ${ }^{6}$ Life Sciences Institute, University of Michigan, Ann Arbor Michigan, 48109
}

\begin{abstract}
Biocatalytic reactions embody many features of ideal chemical transformations, including the potential for impeccable selectivity, high catalytic efficiency, mild reaction conditions and the use of environmentally benign reagents. These advantages have created a demand for new biocatalysts that expand the portfolio of complexity-generating reactions available to synthetic chemists. However, the tradeoff that often exists between the substrate scope of a biocatalyst and its selectivity limits the application of enzymes in synthesis. We recently demonstrated that a flavin-dependent monooxygenase, TropB, maintains high levels of site- and stereoselectivity across a range of structurally diverse substrates. ${ }^{1}$ Herein, we disclose the structural basis for substrate binding in TropB, which performs a synthetically challenging asymmetric oxidative dearomatization reaction with exquisite site- and stereoselectivity across a range of substrates, providing a foundation for future protein engineering and reaction development efforts. Our hypothesis for substrate binding is informed by the first crystal structure of TropB and molecular dynamics simulations with the corresponding computational TropB model and is supported by experimental data. In contrast to canonical type A FADdependent monooxygenases in which substrates bind in a protonated form, our data indicate that the phenolate form of the substrate binds in the active site. Furthermore, the substrate position is controlled through two-point binding of the phenolate oxygen to Arg206 and Tyr239, which are shown to have distinct and essential roles in catalysis. Our studies also demonstrate that Arg206 is involved in the reduction process of the flavin cofactor, suggesting a role in flavin dynamics. Further, QM/MM simulations reveal the interactions that govern the facial selectivity that leads to a highly enantioselective transformation. Thus, the structural origins of the impeccable site- and stereoselectivity observed in reactions of TropB across a range of substrates are elucidated, providing a foundation for future protein engineering and reaction development efforts.
\end{abstract}

\section{Introduction}

The demand for biocatalytic transformations is growing as chemists recognize the advantages of these processes, including high levels of selectivity, low catalyst loadings, environmentally benign reagents and excellent sustainability profiles. ${ }^{2}$ For transformations that pose a challenge to traditional chemical synthesis, there is a greater interest in developing biocatalytic methods. The oxidative dearomatization of phenols to generate quinol products is a reaction that presents a substantial selectivity challenge using traditional chemical methods (see $\mathbf{1}$ to $\mathbf{2}$, Fig. 1). ${ }^{3}$ A suite of $\mathrm{I}(\mathrm{III}), \mathrm{I}(\mathrm{V}), \mathrm{Cu}(\mathrm{I})$ and $\mathrm{Pb}(\mathrm{IV})$ reagents have been developed for oxidative dearomatization. ${ }^{4-8}$ In these reactions, a phenolic substrate is activated as an electrophile, which is intercepted by an oxygen nucleophile in either an intra- or intermolecular fashion. ${ }^{9}$ Achieving high levels of selectivity in this reaction is desirable as site-selectivity is often substrate rather than catalyst-controlled. Further, controlling stereoselectivity under catalytic conditions remains a challenge. ${ }^{5,10-14}$ For example, Maruoka and coworkers recently reported an indane-based organoiodine catalyst that provides $p$-quinol products in up to $84 \%$ enantiomeric excess (ee), the highest stereoselectivity for catalytic oxidative dearomatization reported to date. ${ }^{15}$ Anticipating that a biocatalyst could provide exceptional selectivity and a 
superior sustainability profile, we recently investigated the substrate scope and scalability of a panel of flavindependent monooxygenases that mediate oxidative dearomatization reactions in various secondary metabolite pathways. ${ }^{1}$ In contrast to traditional chemical transformations where site-selectivity is governed by the substrate, selectivity in the flavin-dependent monooxygenase reaction is dictated by the pose of the substrate in the active site as it approaches the active oxidant, C4a-hydroperoxyflavin (12), bound within the active site. ${ }^{16,17} \mathrm{We}$ envisioned that a suite of flavindependent monooxygenases could provide complementary selectivity and redefine the state-of-the-art method for mediating this transformation. Our initial study demonstrated that three biocatalysts, TropB, AzaH, and SorbC, perform well in preparative-scale reactions on a range of substrates while maintaining high levels of siteand stereoselectivity, providing a single product in $94 \%$ to $>99 \%$ ee. ${ }^{1}$ For example, TropB oxidizes non-native substrates such as 3-11, which vary significantly in electronic and steric properties compared to the native TropB substrate 1, yet for each substrate a single product is generated in $\geq 98 \%$ ee (Fig. 1). ${ }^{1}$

We aim to enhance the synthetic utility of these biocatalysts by expanding the substrate scope, developing a suite of catalysts with complementary selectivities, and accessing non-native mechanistic pathways. However, to accomplish these goals and fully realize the synthetic potential of TropB and related enzymes, a structural and mechanistic understanding of this highly selective biocatalyst is required. We solved a crystal structure of TropB, which indicated that the active site is sufficiently spacious to accommodate a range of substrates, but must maintain a conserved binding orientation to achieve high site- and stereoselectivity across an array of substrates. ${ }^{1}$ To provide a detailed understanding of the active site architecture, substrate binding pose, and residues required for catalysis, we carried out molecular dynamics simulations with a corresponding computational enzyme model. Detailed characterization of wild type TropB alongside several variants revealed distinct roles for two amino acids in the active site.

\section{Results and discussion}

Structure of TropB. As a first step toward elucidating the structural basis for selectivity, we solved a $1.7 \AA$ crystal structure of TropB with its flavin adenine dinucleotide (FAD) cofactor bound (Fig. 2A). TropB crystallized as a dimer, consistent with a dimeric form observed by sizeexclusion chromatography (Supplemental Information S2). The large dimer interface $\left(1,244 \AA^{2}\right.$ of buried surface

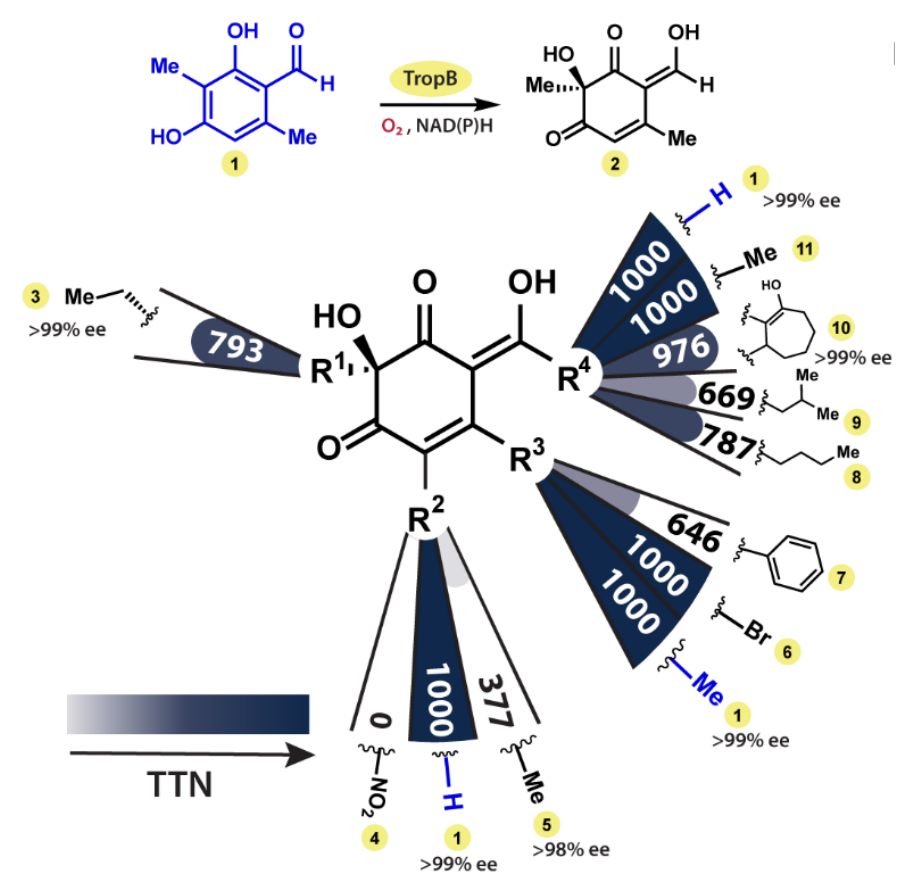

Figure 1. Chemistry of flavin-dependent monooxygenase TropB. ${ }^{1}$ Oxidative phenol dearomatization mediated by TropB in stipitatic acid biosynthesis and substrate scope. Non-native substrates with pertuberation of a single substituent compared to the native substrate $(\mathbf{1}$, blue) were evaluated for reactivity with TropB measured in total turnover numbers (TTNs), maximum TTN $=1000$ in this assay.

area) includes 14 salt bridges and 6 additional hydrogen bonds. The overall structure comprises two domains: (a) FAD binding domain 1, typical type A flavin-dependent monooxygenase with a nucleotide binding site for the adenosine diphosphate (ADP) moiety of the cofactor and (b) catalytic domain 2 with a binding site for the isoalloxazine ring (Supporting Information Figure S7A). ${ }^{16}$ Part of the interdomain loop between $\alpha 4$ (domain 1) and $\beta 4$ (domain 2) is disordered (residues 81-86) (Fig. 2A). Two long helices $(\alpha 11$ and $\alpha 12)$ also connect domains 1 and 2.

A molecule of FAD sits in the narrow interdomain cleft, with a planar isoalloxazine ring that is consistent with the observed yellow color of crystals (Supporting Information Figure S6). FAD contacts the protein through a combination of hydrogen bonds and van der Waals interactions. The adenosine and pyrophosphate groups are bound to TropB as in other type A FADdependent monooxygenases. ${ }^{18}$ The isoalloxazine ring sits in a binding pocket formed by Pro329, His330 and His331 with hydrogen bonds of $\mathrm{O} 4$ and N3 to Ala55, N1 to Ala335 and $\mathrm{O} 2$ to the Cys336 amide (Supporting Information Figure S6). A chloride ion sits in a tight pocket between 

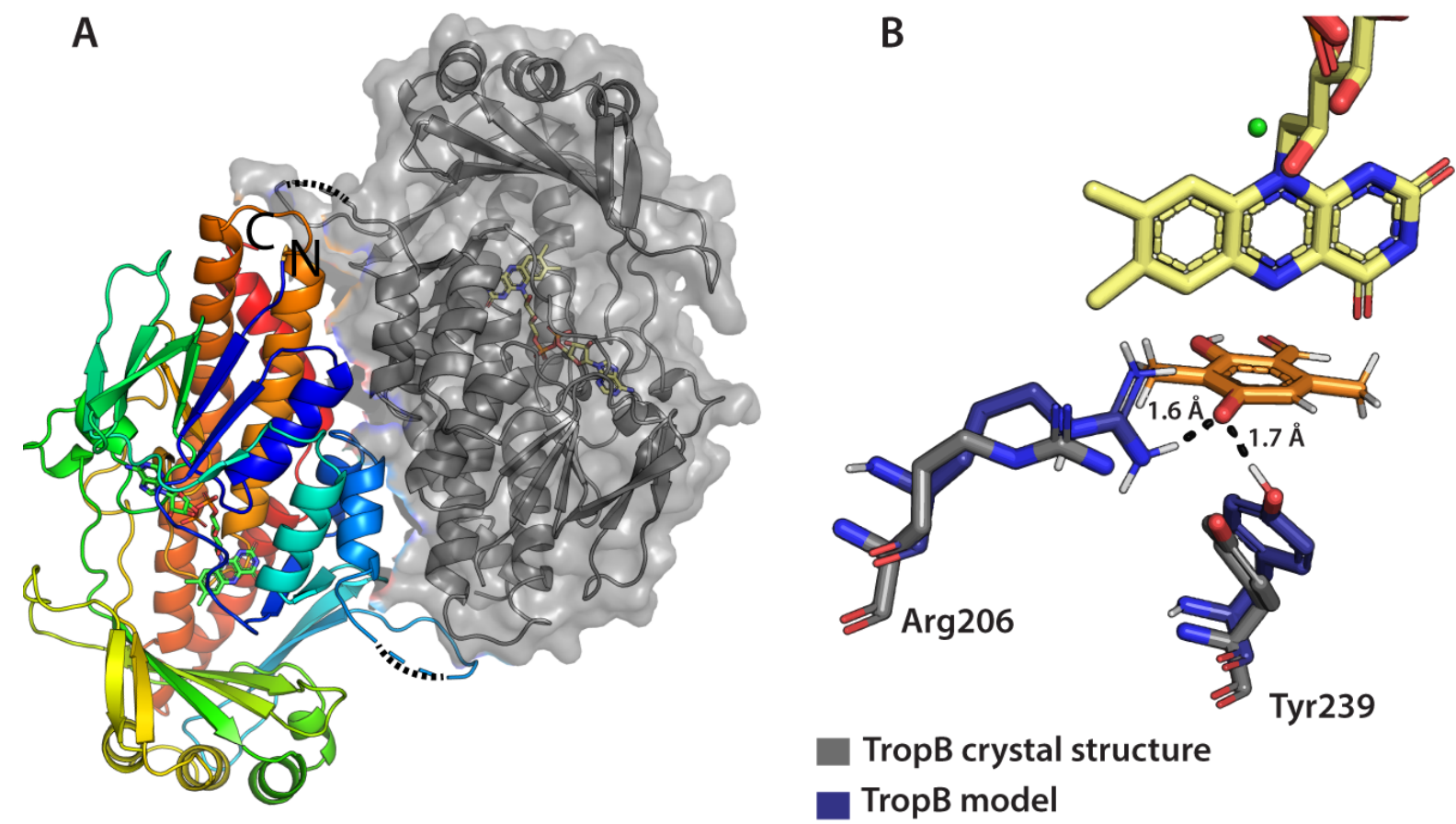

Figure 2. (A) TropB dimer, with monomer 1 colored in rainbow from $\mathrm{N}$ terminus (blue) to $\mathrm{C}$ terminus (red) with disordered residues 81-86 shown as a dashed line and monomer 2 shown in gray; (B) TropB wide-open active site with FAD shown in yellow stick; (C) TropB crystal structure overlaid with the QM/MM model of substrate (1).

the isoalloxazine ring and Pro329, Gly332 and Gly334, as in other FAD class A monooxygenases (Supporting Information Figure S7B). ${ }^{19-21}$

Crystallized in the absence of substrate, a large cavity adjacent to the isoalloxazine ring is anticipated to be the site of substrate binding. This site is defined by several side chains (Arg206, Leu226, Met228, His235, Ile237, Tyr239, Ala250, Phe252, Pro329, His330, His331, and Tyr397), some of which are conserved in other type A flavin monooxygenases. ${ }^{19}$ One molecule of hexanediol, a component of the crystallization solution, was bound in the cavity, based on well-defined electron density (Supporting Information Figure S8).

As crystallization with the flavin cofactor in the oxidized state should permit formation of a dead-end substrate complex, significant effort was dedicated to obtaining crystals of a TropB ternary complex with FAD and the native substrate, 3-methylorcinaldehyde (1). Crystal soaking and co-crystallization experiments with 1 or structural analogs of $\mathbf{1}$ resulted in maps with new electron density in the active site cavity; however, despite extensive experimentation none of the conditions explored led to well-defined density for a single orientation of the substrate or to the full displacement of hexanediol from the cavity (Supporting Information Figure S8). Although full occupancy of a TropB ternary complex was not captured, crystallization in the presence of $\mathbf{1}$ induced a change in position of the cofactor isoalloxazine ring, consistent with flavin dynamics of type A flavin-dependent monooxygenases. ${ }^{22,23}$ Substrate binding in type A flavindependent monooxygenases triggers flavin reduction by inducing a "flavin-out" conformation, in which the flavin is accessible to the reduced nicotinamide adenine dinucleotide phosphate (NADPH) or nicotinamide adenine dinucleotide (NADH) cofactor (Supporting Information Figure S9) ${ }^{23-25}$ The TropB crystal structure obtained in the absence of substrate depicts the oxidized cofactor in the "flavin-in" conformation (Supporting Information Figure S8), whereas the structure in the presence of substrate indicated partial occupancy of a flavin-out conformation (Supporting Information Figure S9). This change in flavin position upon binding of substrate adjacent to the flavin-in position would allow for reduction of FAD to $\mathrm{FADH}_{2}$ by an external reductant (NADH or NADPH), consistent with other class A flavin dependent monooxygenases (Supporting Information Figure S9). ${ }^{23-25}$

Substrate binding and catalysis. Arg206 and Tyr239 are the only amino acids in the TropB active site that possess relevant functional groups and are near the flavin cofactor. In the absence of clear density to illustrate the contacts of the native substrate (1), we sought to draw an analogy to 
substrate complexes of other class A flavin-dependent monooxygenases of known structure. TropB has low sequence identity to other class A flavin-dependent monooxygenases, except for SalA $(31 \%) ;^{18}$ nonetheless, structural features involved in either substrate binding or catalysis remain constant across enzymes in this class. In p-hydroxyl benzoate hydroxylase (PHBH) an hydrogen bond network composed of Tyr201, Tyr385, His 72 and a conserved water molecule is responsible for substrate binding and further catalysis. ${ }^{17}$ This network is absent in TropB; however, Tyr239 and Arg206 are within hydrogen bonding distance of the substrate in the TropB active site. Additionally, the Arg206 guanidinium group is in a position analogous to the Arg220 guanidinium group in PHBH. This is also the case for other class A monooxygenases, PHHY (Tyr289, Arg287), ${ }^{26} \mathrm{MHBH}$ $\left(\right.$ Tyr271, Arg262) ${ }^{27}$ and SalA (Arg190). ${ }^{18}$ This led us to hypothesize that Tyr239 and Arg206 might be involved in substrate binding and perhaps catalysis.

A set of TropB variants was generated to probe the role of Tyr239 and Arg206 in substrate binding. Arg206 was substituted with either Glu or Gln and Tyr239 was replaced with Phe (Supporting Information Table 2, Figure S23). TropB R206E, TropB R206Q, and TropB Y239F produced no detectable product by UPLC-PDA analysis under conditions where the wild type TropB led to complete consumption of the substrate. These results indicate that Arg206 and Tyr239 each have a critical role in achieving productive catalysis.

Having established that TropB R206E, TropB R206Q, and TropB Y239F produce no detectable product with the native substrate, 3-methylorcinaldehyde (1), we sought to identify which step of the catalytic cycle was disrupted. Measurement of the $\mathrm{K}_{\mathrm{d}}$ values with 1 by UVVis revealed a $\mathrm{K}_{\mathrm{d}}$ of $21 \pm 2 \mu \mathrm{M}$ for wild-type TropB and $32 \pm 4 \mu \mathrm{M}$ for TropB R206Q. Substituting Arg206 or Tyr239 with an amino acid that cannot act as a hydrogen bond donor led to a significant increase in $\mathrm{K}_{\mathrm{d}}$ values (Fig. 3 ), suggesting that hydrogen bond donors at positions 206 and 239 are critical for substrate binding. Next, we investigated whether NADPH can reduce FAD to $\mathrm{FADH}_{2}$ in each TropB variant by UV-Vis. Consumption of NADPH was observed for wild type TropB and at a slower rate for TropB Y239F. No detectable depletion of NADPH was observed for TropB R206Q. To further probe cofactor reduction, the enzyme concentration was increased 200fold. At the higher enzyme concentration, we observed consumption of the oxidized cofactor after 3 hours. To generate a detectable amount of the reduced FAD to confirm the involvement of the residue in the reduction step, anaerobic reduction of TropB R206Q in the presence of substrate and NADH was monitored over the span of $3.3 \mathrm{~h}$. The FAD was fully reduced at a significantly slower rate in TropB R206Q than in the wild type TropB. (Fig. 3 and Supporting Information Fig. S32). Previously Ballou and coworkers demonstrated that the corresponding Arg residue (Arg220) in PHBH, a well-studied type A flavindependent monooxygenase, is involved in the flavin dynamics required for FAD to toggle from the "in" conformation to the "out" conformation for reduction of FAD to $\mathrm{FADH}_{2}$ by stabilizing the "out" conformation. ${ }^{23}$ To test the ability of each TropB variant to engage molecular oxygen to form the labile C4a-hydroperoxyflavin intermediate, the hydrogen peroxide concentration was measured in samples containing each TropB variant with $2.5 \mathrm{mM}$ substrate and $\mathrm{NADPH}$, generating a readout on the
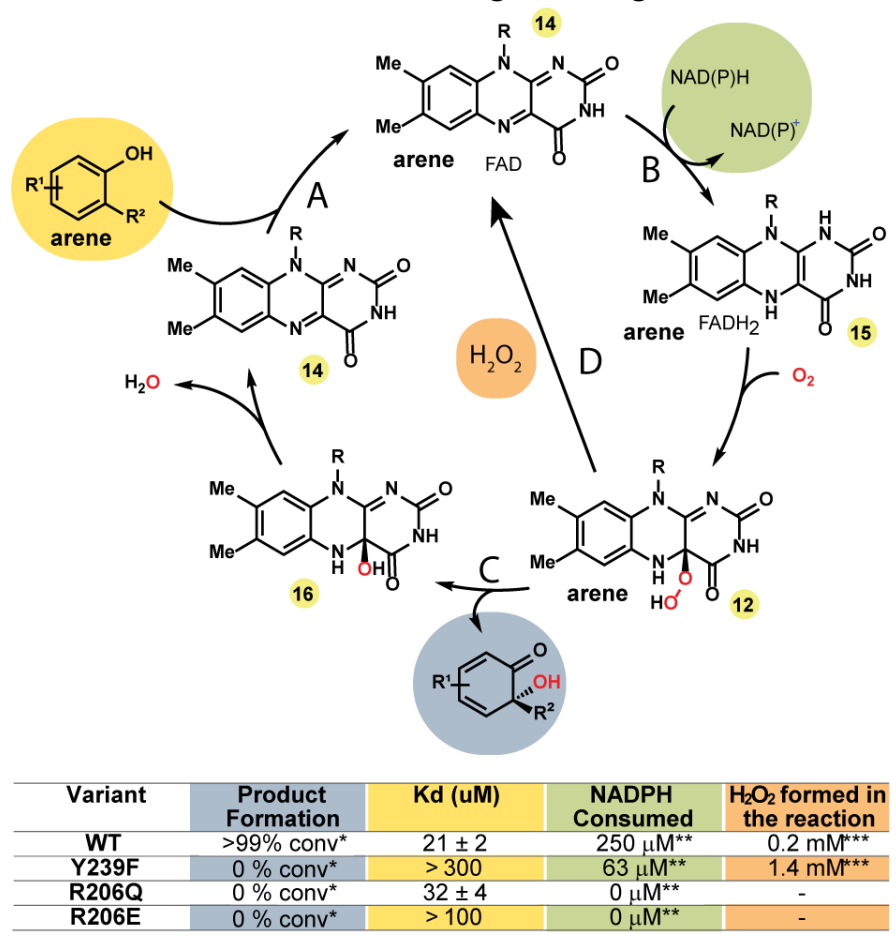

Figure 3. Characterization of WT TropB and TropB variants. (A) Substrate binding of substrate 1 to TropB wild-type;(B) NADPH depletion upon substrate 1 binding; (C) Product formation after reaction with substrate $\mathbf{1}$ with the respective enzyme; (D) Peroxide formation after reaction with substrate 1. *Reaction conditions: $2.5 \mathrm{mM}$ Substrate 1, 2.5 TropB variant, $1 \mathrm{mM} \mathrm{NADP}^{+}, 5 \mathrm{mM}$ glucose-6-phosphate (G6P), 1 u mL ${ }^{-1}$ glucose-6-phophate dehydrogenase (G6PDH), $50 \mathrm{mM}$ potassium phosphate buffer, $\mathrm{pH} 8.0,30^{\circ} \mathrm{C}, 1 \mathrm{~h} .0 \%$ conversion indicates that no substrate consumption or product formation was detected. **Reaction Conditions: $100 \mathrm{nM}$ TropB variant, $250 \mu \mathrm{M} \mathrm{NADPH}$, and $125 \mu \mathrm{M}$ substrate $\mathbf{1}$ monitored at 362 $\mathrm{nm}$ over $1,000 \mathrm{sec} .{ }^{* * *}$ Assay conditions: $2.5 \mu \mathrm{M}$ HRP, 0.75 $\mathrm{mM}$ 4-amino antipyrine, 2,4,6-tribromo-3-hydroxybenzoic acid dye $(0.02 \% \mathrm{w} / \mathrm{v})$, phosphate buffer $\mathrm{pH} 8.0(100 \mathrm{mM})$ measured at $510 \mathrm{~nm}$. 
level of peroxide uncoupling (see 12 to 14, Fig. 3D). In this assay, relatively low levels of $\mathrm{H}_{2} \mathrm{O}_{2}$ were measured $(0.2 \mathrm{mM})$ with wild type TropB, whereas, significantly higher concentrations of $\mathrm{H}_{2} \mathrm{O}_{2}$ were present in reactions with TropB Y239F (1.4 mM), indicating a higher level of uncoupling. In reactions with TropB (R206E and R206Q), trace amounts of $\mathrm{H}_{2} \mathrm{O}_{2}$ were detected $(0.03 \mathrm{mM})$, which did not increase over the course of the reaction, further indicating that these substitutions hindered flavin reduction. Characterization of the Arg206 and Tyr239 variants supports the role of these two amino acids in substrate binding. These results indicate that $\operatorname{Arg} 206$ is also essential for FAD reduction to $\mathrm{FADH}_{2}$ and that Tyr239 stabilizes the substrate in a position suitable for rapid reaction with the labile $\mathrm{C} 4 \mathrm{a}$-hydroperoxyflavin intermediate. Moreover, the variants TropB R206Q and TropB Y239F were crystallized (Supporting Information Table S2). In the crystal structure of TropB R206Q, Gln206 is ordered and within hydrogen bond distance of Gly207. In the TropB Y239F structure, Phe239 maintains the same position as Tyr239 in the wild type protein (Supporting Information, Figure S11).

Computational approaches to elucidate substrate binding pose. To provide additional insight on the pose of the native substrate $\mathbf{1}$ in the active site, we turned to computational analysis. Several enzyme-substrate models with the flavin cofactor as either FAD (14) or C4ahydroperoxyflavin (12) were evaluated with ligandprotein docking methods and molecular dynamics (MD) simulations. It was hypothesized by the Cox group that the substrate undergoes a deprotonotation by His235, as deprotonation is a common step for other class A flavindependent monooxygenases. ${ }^{28}$ It is generally accepted that type A FAD-dependent monooxygenases require a catalytic base for deprotonation of phenolic substrates in order to obtain a sufficiently nucleophilic reactant. ${ }^{16,29,30}$ Deprotonation is thought to occur prior to flavin reduction or during substrate hydroxylation. ${ }^{17}$ In the case of TropB, His 235 is a candidate catalytic base, in principle; however, the crystal structure indicates that His 235 is too far from the substrate to perform a deprotonation. Additionally, docking studies indicated that all of the models with the substrate in the neutral form failed to generate interactions that could activate the substrate through deprotonation or to orient the substrate in a pose that would allow for a reaction with the site- and stereoselectivity necessary to generate the observed product 2 .

The apparent lack of a basic residue coupled with unproductive docking studies with the neutral form of the substrate led us to hypothesize that the substrate binds as the phenolate. If the substrate binds in the deprotonated form, a base would not be required for catalysis as previously suggested (Fig. 4). ${ }^{16,28,31,32}$ Flexible docking with the substrate in the anionic form led to a favorable, low-energy pose consistent with the known site- and stereoselectivity of the reaction. This docking indicated that both Arg206 and Tyr239 hydrogen bond with the phenolate oxygen of $\mathbf{1 3}$ (Figs. 2B and 4). The pose showed a significant change in the location of Arg206 from its position in the crystal structure, while the movement of Tyr239 was minimal (Fig. 2B). The displacement of Arg206 is reasonable given that the enzyme was crystallized with the flavin in the oxidized "in" state and must go through several catalytic steps before reaching the C4a-hydroperoxyflavin state (12, see Fig. 3). The charge distribution in the molecular mechanics (MM) force field delocalizes the negative charge on the substrate in
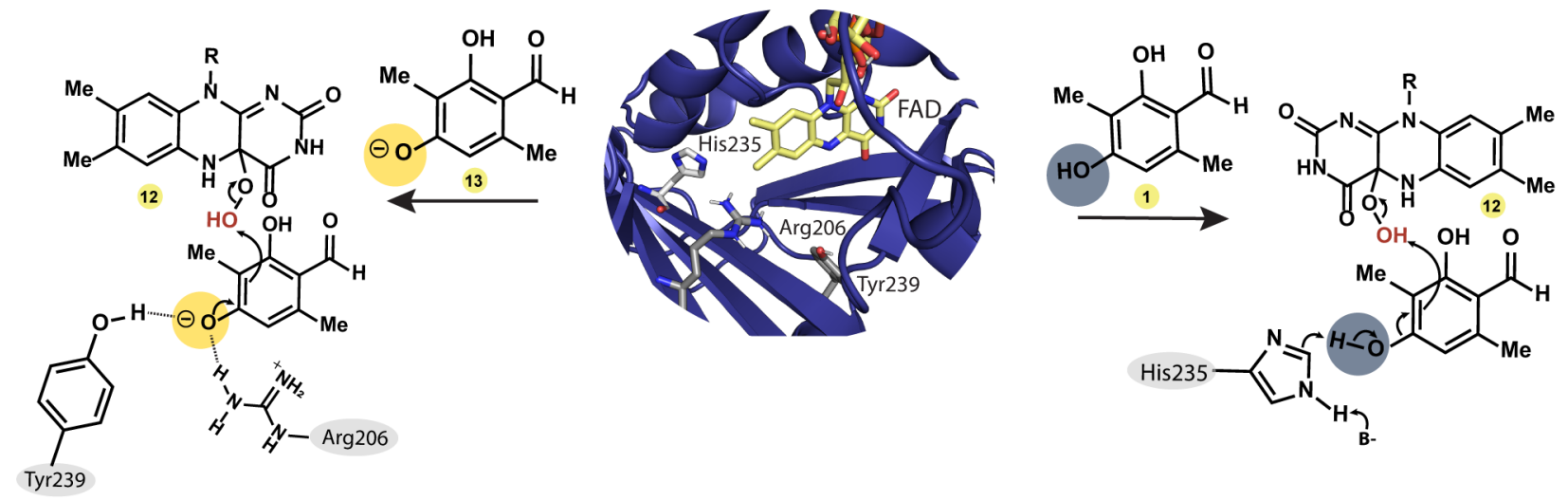

Figure 4. Hypotheses for substrate activation and positioning. The mechanism on the right requires deprotonation of the substrate in the active site, which has been proposed to be performed by His $235 .{ }^{19}$ The mechanism proposed on the left relies on binding of the anionic form of the substrate, including interactions with Arg206 and Tyr239 consistent with our experimental findings. The C4a-hydroperoxoflavin $\mathrm{O}_{\text {distal }}$ is highlighted in red. 
accordance with gas phase and solvated QM calculations and was not concentrated on the phenolate oxygen. This indicates that the interaction with Arg206 and Tyr239 was not driven by an unphysical electrostatic interaction that could arise if the charges were overly localized by standard charge distribution methods.

To characterize enzyme-substrate contacts, several MD simulations of the identified docking pose were performed. Specifically, models with different tautomeric states of His 235 , His 330 and His 331 were constructed since 1) the tautomeric states of His residues are not directly observed in crystal structures 2) these states can be challenging to accurately predict and 3) these states will affect the active site interactions and dynamics. During each of the 25 nanosecond (ns) simulations, the substrate exhibited only minimal fluctuations ( 0.24 to 0.65 $\AA$ root mean square deviation [rmsd] from average atomic position) remaining in a bound position (averaging 5.2 to $6.8 \AA$ for the $\mathrm{O}_{\text {distal }}-\mathrm{C} 3$ distance, Supporting Information Figure S36 and Table S3). A reactive configuration was observed in $1.3 \%$ of the snapshots as strictly defined by (1) an $\mathrm{O}_{\text {distal }}-\mathrm{C} 3$ distance less than $4 \AA$ and, (2) an $\mathrm{O}_{\text {distal }}-\mathrm{C} 3$ $\mathrm{C} 3$ methyl angle between $60^{\circ}$ and $115^{\circ}$, (Supporting Information, Figure S35). All of these snapshots showed a hydrogen bond between the anionic substrate $\mathbf{1 3}$ and Arg206, and approximately half of the snapshots also showed a hydrogen bond with Tyr239, supporting the relevance of this interaction. MD simulations often show only a small fraction of snapshots which have all of the elements that would favor a chemical reaction. ${ }^{33,34}$ One of the Arg206 terminal amine groups hydrogen bonded to the phenolate oxygen throughout the simulation (average $\mathrm{O}-\mathrm{H}$ distance $2.0 \AA, 88 \%$ of frames $<2.4 \AA$ ). Interestingly, the phenolic proton of Tyr239 formed a tighter hydrogen bond as the simulation progressed. The average distance for this hydrogen bond was $2.7 \AA$ over 24 ns but averaged $2.0 \AA$ in the final $10 \mathrm{~ns}$ with $90 \%$ of the snapshots showing a distance less than $2.4 \AA$ (Supporting information Figure S35). Thus, MD simulations with a classical force field suggest that both Arg206 and Tyr239 form hydrogen bonding interactions with the substrate, which appear to be the dominant factor in binding the substrate while the cofactor is in the C4a-hydroperoxyflavin state.

In order to investigate the interactions between the substrate and the active site environment with a more accurate description, MD simulations were also performed using a QM/MM representation. ${ }^{35}$ The substrate maintained hydrogen bonding interactions with both Tyr239 and Arg206 throughout these simulations. In addition, the QM/MM calculations showed significant charge transfer of -0.35 electrons from the substrate to the enzyme environment, leaving the substrate with a total charge of only -0.65 electrons in the Michaelis complex. The transfer of charge resulted in increased electrostatic interactions and thus a more tightly bound substrate.

The combined knowledge from the TropB crystal structure and subsequent $\mathrm{MD}$ suggests that the anionic form of the substrate, phenolate $\mathbf{1 3}$, is the predominant form under the reaction conditions and that the negative charge is stabilized through interactions with both Arg206 and Tyr239, thus positioning the substrate for a productive reaction with the $\mathrm{C} 4 \mathrm{a}-$ hydroperoxyflavin cofactor (12, Fig. 4) and leading to a highly site- and stereoselective transformation.

To interrogate this hypothesis, we first investigated the protonation state of the substrate under the reaction conditions, specifically the optimum $\mathrm{pH} 8 .^{28}$ The protonation state of the native substrate (1) was monitored by UV absorbance, as the phenolate absorbs at $334 \mathrm{~nm}$, whereas, the protonated form is characterized by a maximum absorbance at $292 \mathrm{~nm}$ (Supporting Information Figure S28). This indicates that, under the reaction conditions at $\mathrm{pH} 8$, the predominant form of the substrate is the anionic form (13) with an experimentally determined pKa of 7.1. To experimentally test the proposal that $\mathrm{H} 235$ is necessary for catalysis (Fig. 4), ${ }^{28}$ TropB H235A was generated. Substitution of His 235 with Ala produced a catalytically active TropB variant (10\% conversion, Supporting Information Figures S19 and S23). To probe the role of a neighboring His residue, His330 was substituted with Ala, affording an active variant capable of $11 \%$ conversion of substrate to dearomatized product. Since these residues are close together, one could imagine His235 and His330 having redundant function as the requisite base. Thus, a double substitution was performed to generate $\mathrm{H} 235 \mathrm{~A} / \mathrm{H} 330 \mathrm{~A}$ and the variant remained active $(10 \%$ conversion, Supporting information S22 and Supporting Information S23), These results are consistent with the anionic form of the substrate binding, eliminating the need for a basic residue in the active site for substrate deprotonation.

Computational insights on facial selectivity. While QM/MM simulations indicate that hydrogen bonding of Arg206 and Tyr239 with the phenolate form of the substrate is the dominant intermolecular interaction, these interactions alone are insufficient to be the basis of facial selectivity. Additional interactions between substrate and TropB were observed in the simulations. Both hydrophobic C3 and C6 methyl groups of substrate $\mathbf{1}$ interacted with hydrophobic residues. The C6 methyl group was near Ala55 and the phenyl group of Phe119. In 

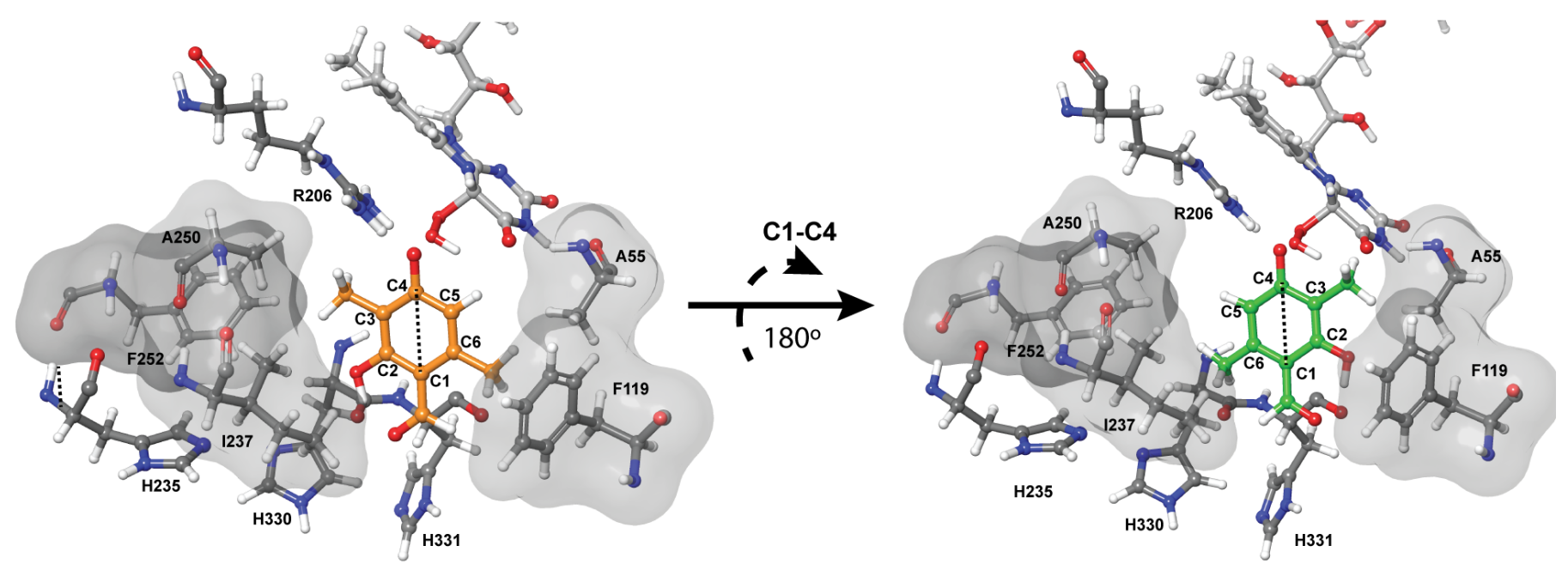

Figure 5. QM/MM simulation of substrate (1) in the active site to illustrate facial selectivity. (A) Substrate (1) in orange bound to the active-site, showing favorable contacts with Ala250 and Ile237; (B) $180^{\circ}$ degree rotation along the C1-C4 axis of native substrate (1) highlighted in magenta, showing a potential steric clash with Tyr239.

contrast, the C2-hydroxyl group was positioned in a solvated environment, hydrogen bonding exclusively with the aldehyde moiety. If the opposite face of the substrate were presented to the $\mathrm{C} 4 \mathrm{a}-\mathrm{C} y d r o p e r o x y f l a v i n$ factor (12) by rotating along the $\mathrm{C} 1-\mathrm{C} 4$ axis, the $\mathrm{C} 2$ hydroxyl group would occupy an unsuitable hydrophobic region and the C6 methyl group would be in a hydrophilic space, placing these two groups in uncomplimentary environments (Fig. 5 and Supporting Information Figure S46). Similarly, the C3 methyl group makes hydrophobic contacts with Phe252, Ala250 and Ile237. If the substrate rotated about the C1-C4 axis, however, this methyl group would sit near the backbone carbonyl of Gly53 and the hydroperoxy group of the flavin. Thus, this region would be an energetically unfavorable position for the hydrophobic $\mathrm{C} 3$ methyl group if the substrate presented the opposite face to the cofactor. The bulkier methyl group would also clash with Tyr239, disrupting the hydrogen bond with the substrate. Thus, we conclude that the facial selectivity in the hydroxylation of substrate $\mathbf{1}$ in TropB arises from the amphipathic active site environment as illustrated in Figure 5.

Through structural insights gained from both the crystal structure and molecular dynamics simulations, we have uncovered the origins of the exquisite site- and stereoselectivity exhibited in reactions of TropB with a breadth of substrates. A crystal structure of TropB has enabled computational studies that support binding of the anionic form of the substrate, which is further supported by experimental data. Molecular dynamics simulations and characterization of TropB variants demonstrated that the phenolate substrate has significant interactions with both Arg206 and Tyr239, controlling the pose of the substrate within the active site and the selectivity observed in subsequent reactions. The excellent stereoselectivity observed in reactions with TropB can be attributed to pronounced facial selectivity in substrate binding. These findings clearly illustrate the structural features that control the site- and stereoselectivity in the complexitygenerating reaction mediated by TropB, critical for future protein engineering and reaction development efforts with TropB and related enzymes.

\section{Author Information \\ Corresponding Author}

*arhardin@umich.edu

ORCID Alison R. H. Narayan: 0000-0001-8290-0077

Notes: The authors declare no competing financial interest.

\section{Acknowledgements}

This research was supported by funds from the University of Michigan Life Sciences Institute, the National Institutes of Health R35 GM124880 (to A.R.H.N.) and R01 DK042303 (to J.L.S.), and NSF CHE 1506273 (to C.L.B.). A.R.B. and A.L.L. were supported by a Graduate Assistance of Areas in National Need Training Grant (GAANN P200A150164), A.R.B. and S.A.B.D. were supported by a National Institutes of Health Chemistry Biology Interface Training Grant (T32 GM008597), and A.R.B was also supported by a Rackham Merit Fellowship. GM/CA@APS is supported by the NIH National Institute of General Medical Sciences (AGM12006) and National Cancer Institute (ACB-12002). We 
are grateful to Professor Bruce Palfey for insightful discussions and the use of equipment.

\section{References}

(1) Baker Dockrey, S. A.; Lukowski, A. L.; Becker, M. R.; Narayan, A. R. H. Biocatalytic Site- and Enantioselective Oxidative Dearomatization of Phenols. Nat. Chem. 2017, 10, 119-125.

(2) Sheldon, R. A.; Woodley, J. M. Role of Biocatalysis in Sustainable Chemistry. Chem. Rev. 2018, 118, 801-838.

(3) Roche, S. P.; Porco, J. A. Dearomatization Strategies in the Synthesis of Complex Natural Products. Angew. Chem. Int. Ed. Engl. 2011, 50, 4068-4093.

(4) Roche, S. P.; Porco, J. A. Dearomatization Strategies in the Synthesis of Complex Natural Products. Angew. Chemie - Int. Ed. 2011, 50, 4068-4093.

(5) Zhu, J.; Grigoriadis, N. P.; Lee, J. P.; Porco, J. A. Synthesis of the Azaphilones Using Copper-Mediated Enantioselective Oxidative Dearomatization. J. Am. Chem. Soc. 2005, 127, 9342-9343.

(6) Wang, W. X.; Kusari, S.; Laatsch, H.; Golz, C.; Kusari, P.; Strohmann, C.; Kayser, O.; Spiteller, M. Antibacterial Azaphilones from an Endophytic Fungus, Colletotrichum Sp. BS4. J. Nat. Prod. 2016, 79, 704-710.

(7) Yang, Q.; Draghici, C.; Njardarson, J. T.; Li, F.; Smith, B. R.; Das, P. Evolution of an Oxidative Dearomatization Enabled Total Synthesis of Vinigrol. Org. Biomol. Chem. 2014, 12, 330-344.

(8) Shiao, H. Y.; Hsieh, H. P.; Liao, C. C. First Total Syntheses of $( \pm)$-Annuionone B and $( \pm)$ - Tanarifuranonol. Org. Lett. 2008, 10, 449-452.

(9) Sun, W.; Li, G.; Hong, L.; Wang, R. Asymmetric Dearomatization of Phenols. Org. Biomol. Chem. 2016, 14, 2164-2176.

(10) Uyanik, M.; Yasui, T.; Ishihara, K. Hydrogen Bonding and Alcohol Effects in Asymmetric Hypervalent Iodine Catalysis: Enantioselective Oxidative Dearomatization of Phenols. Angew. Chemie - Int. Ed. 2013, 52, 9215-9218.

(11) Bosset, C.; Coffinier, R.; Peixoto, P. A.; El Assal, M.; Miqueu, K.; Sotiropoulos, J. M.; Pouységu, L.; Quideau, S. Asymmetric Hydroxylative Phenol Dearomatization Promoted by Chiral Binaphthylic and Biphenylic Iodanes. Angew. Chem. Int. Ed. 2014, 53, 9860-9864.

(12) G. Billek, J. S. and F. W. Die Dienon-PhenolUmlgerung Des 2,2-Diacetoxy-Cyclohexa-3,5-Dienons-1. Tetrahedron 1962, 18, 909-915.

(13) Volp, K. A.; Harned, A. M. Chiral Aryl Iodide Catalysts for the Enantioselective Synthesis of ParaQuinols. Chem. Commun. 2013, 49, 3001.

(14) Boppisetti, J. K.; Birman, V. B. Asymmetric
Oxidation of O- Alkylphenols with Chiral 2- ( oIodoxyphenyl ) -Oxazolines. Org. Lett 2009, 11, 12211223.

(15) Hashimoto, T.; Shimazaki, Y.; Omatsu, Y.; Maruoka, K. Indanol-Based Chiral Organoiodine Catalysts for Enantioselective Hydrative Dearomatization. Angew. Chem. Int. Ed. 2018, 57, 7200-7204.

(16) Huijbers, M. M. E.; Montersino, S.; Westphal, A. H.; Tischler, D.; Van Berkel, W. J. H. Flavin Dependent Monooxygenases. Arch. Biochem. Biophys. 2014, 544, 2 17.

(17) Palfey, B. A.; McDonald, C. A. Control of Catalysis in Flavin-Dependent Monooxygenases. Arch. Biochem. Biophys. 2010, 493, 26-36.

(18) Uemura, T.; Kita, A.; Watanabe, Y.; Adachi, M.; Kuroki, R.; Morimoto, Y. The Catalytic Mechanism of Decarboxylative Hydroxylation of Salicylate Hydroxylase Revealed by Crystal Structure Analysis at $2.5 \AA$ Resolution. Biochem. Biophys. Res. Commun. 2016, 469, 158-163.

(19) Montersino, S.; Orru, R.; Barendregt, A.; Westphal, A. H.; Van Duijn, E.; Mattevi, A.; Van Berkel, W. J. H. Crystal Structure of 3-Hydroxybenzoate 6-Hydroxylase Uncovers Lipid-Assisted Flavoprotein Strategy for Regioselective Aromatic Hydroxylation. J. Biol. Chem. 2013, 288, 26235-26245.

(20) Ryan, K. S.; Howard-Jones, A. R.; Hamill, M. J.; Elliott, S. J.; Walsh, C. T.; Drennan, C. L. Crystallographic Trapping in the Rebeccamycin Biosynthetic Enzyme RebC. Proc. Natl. Acad. Sci. U.S.A. 2007, 104, 1531115316.

(21) van Berkel, W. J. H.; Kamerbeek, N. M.; Fraaije, M. W. Flavoprotein Monooxygenases, a Diverse Class of Oxidative Biocatalysts. J. Biotechnol. 2006, 124, 670-689. (22) Ballou, D. P.; Entsch, B.; Cole, L. J. Dynamics Involved in Catalysis by Single-Component and TwoComponent Flavin-Dependent Aromatic Hydroxylases. Biochem. Biophys. Res. Commun. 2005, 338, 590-598.

(23) Moran, G. R.; Entsch, B.; Palfey, B. A.; Ballou, D. P. Evidence for Flavin Movement in the Function of PHydroxybenzoate Hydroxylase from Studies of the Mutant Arg220Lys. Biochemistry 1996, 35, 9278-9285.

(24) Gatti, D. L.; Palfey, B. a; Lah, M. S.; Entsch, B.; Massey, V.; Ballou, D. P.; Ludwig, M. L. The Mobile Flavin of 4-OH Benzoate Hydroxylase. Science 1994, 266, 110-114.

(25) Schreuder, H. A.; Mattevi, A.; Obmolova, G.; Kalk, K. H.; Hol, W. G. J.; van der Bolt, F. J. T.; van Berkel, W. J. H. Crystal Structures of Wild-Type p-Hydroxybenzoate Hydroxylase Complexed with 4-Aminobenzoate, 2,4Dihydroxybenzoate, and 2-Hydroxy-4-Aminobenzoate 
and of the Tyr222Ala Mutant Complexed with 2-Hydroxy4-Aminobenzoate. Evidence for a Proton Channel and a Ne. Biochemistry 1994, 33, 10161-10170.

(26) Enroth, C.; IUCr. High-Resolution Structure of Phenol Hydroxylase and Correction of Sequence Errors. Acta Crystallogr. Sect. D Biol. Crystallogr. 2003, 59, 1597-1602.

(27) Hiromoto, T.; Fujiwara, S.; Hosokawa, K.; Yamaguchi, H. Crystal Structure of 3-Hydroxybenzoate Hydroxylase from Comamonas Testosteroni Has a Large Tunnel for Substrate and Oxygen Access to the Active Site. J. Mol. Biol. 2006, 364, 878-896.

(28)Abood, A.; Al-Fahad, A.; Scott, A.; Hosny, A. E.-D. M. S.; Hashem, A. M.; Fattah, A. M. A.; Race, P. R.; Simpson, T. J.; Cox, R. J. Kinetic Characterisation of the FAD Dependent Monooxygenase TropB and Investigation of Its Biotransformation Potential. RSC $A d v$. 2015, 5, 49987-49995.

(29) Montersino, S.; Orru, R.; Barendregt, A.; Westphal, A. H.; Van Duijn, E.; Mattevi, A.; Van Berkel, W. J. H. Crystal Structure of 3-Hydroxybenzoate 6-Hydroxylase Uncovers Lipid-Assisted Flavoprotein Strategy for Regioselective Aromatic Hydroxylation. J. Biol. Chem. 2013, 288, 26235-26245.

(30) Kobayashi, J.; Yoshida, H.; Yagi, T.; Kamitori, S.; Hayashi, H.; Mizutani, K.; Takahashi, N.; Mikami, B. Role of the Tyr270 Residue in 2-Methyl-3-Hydroxypyridine-5Carboxylic Acid Oxygenase from Mesorhizobium Loti. $J$. Biosci. Bioeng. 2017, 123, 154-162.

(31) Crozier-Reabe, K.; Moran, G. R. Form Follows Function: Structural and Catalytic Variation in the Class a Flavoprotein Monooxygenases. Int. J. Mol. Sci. 2012, 13, 15601-15639.

(32) Abood, A.; Al-Fahad, A.; Scott, A.; Hosny, A. E.-D. M. S.; Hashem, A. M.; Fattah, A. M. a.; Race, P. R.; Simpson, T. J.; Cox, R. J. Kinetic Characterisation of the FAD Dependent Monooxygenase TropB and Investigation of Its Biotransformation Potential. RSC $A d v$. 2015, 5, 49987-49995.

(33) Wymore, T.; Hempel, J.; Cho, S. S.; MacKerell, A. D.; Nicholas, H. B.; Deerfield, D. W. Molecular Recognition of Aldehydes by Aldehyde Dehydrogenase and Mechanism of Nucleophile Activation. Proteins Struct. Funct. Bioinforma. 2004, 57, 758-771.

(34) Rod, T. H. and Brooks, C. L. How Dihydrofolate Reductase Facilitates Protonation of Dihydrofolate. J. Am.

Chem. Soc. 2003, 125, 8718-8719.

(35) Gaus, M.; Cui, Q.; Elstner, M. DFTB3: Extension of the Self-Consistent-Charge Density-Functional TightBinding Method (SCC-DFTB). J. Chem. Theory Comput. 2011, 7, 931-948. 